\title{
The mechanism of polydiacetylene blue-to-red transformation induced by antimicrobial peptides
}

\author{
Johann Nuck, ${ }^{1}$ Kaori Sugihara*1,2 \\ ${ }^{1}$ Department of Physical Chemistry, University of Geneva, Quai Ernest Ansermet 30, 1211 Geneva 4, Switzerland \\ ${ }^{2}$ Institute of Industrial Science, The University of Tokyo, 4-6-1 Komaba Meguro-Ku, Tokyo 153-8505, Japan \\ * corresponding author: kaori.sugihara@unige.ch
}

\section{Table of Contents}

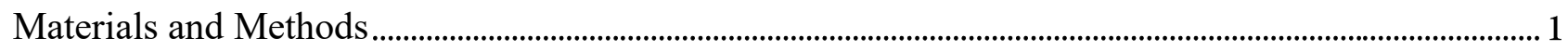

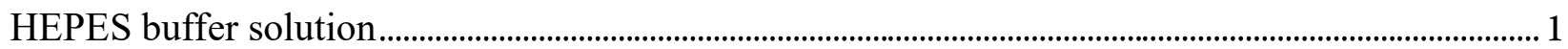

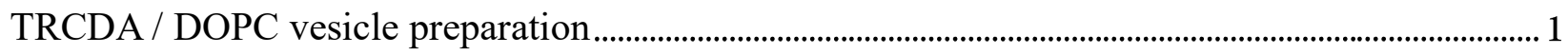

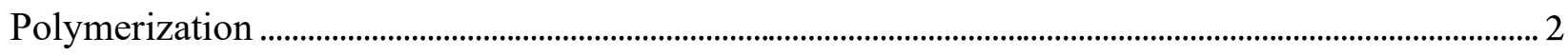

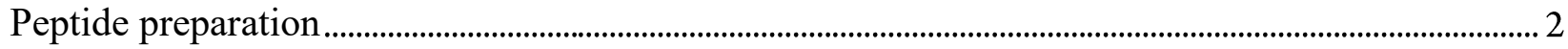

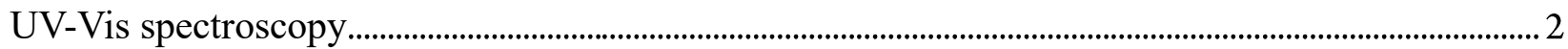

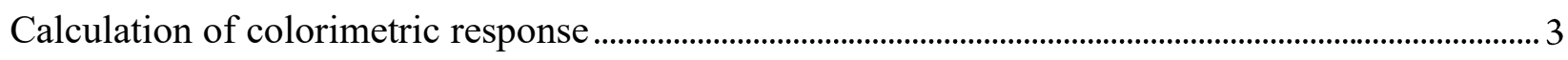

TRCDA/DOPC sample preparation for Figure 1B,D-G and Figure 3 …….............................................. 3

TRCDA sample preparation for Figure 1H,I _................................................................................................. 6

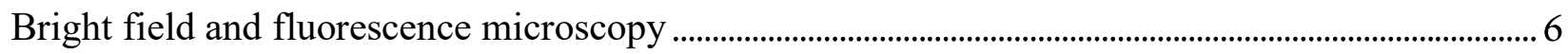

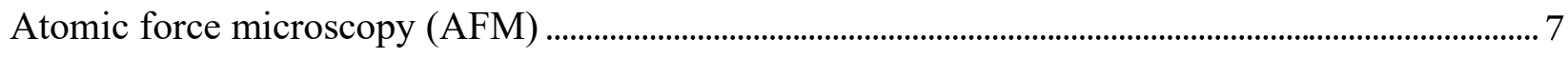

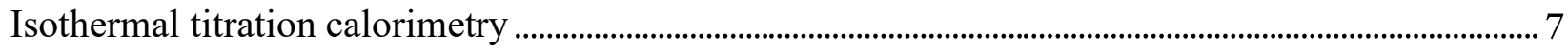

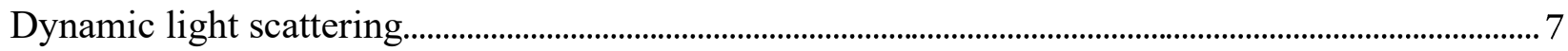

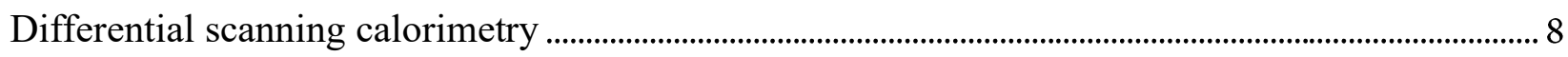

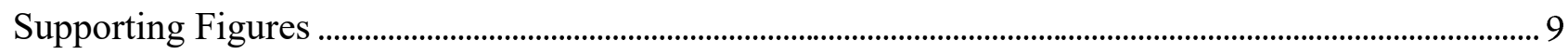




\section{Materials and Methods}

\section{HEPES buffer solution}

The experiments were performed in physiological buffer solution prepared with $10 \mathrm{mM}$ 4-(2hydroxyethyl)piperazine-1-ethanesulfonic acid (HEPES, CAS RN: 7365-45-9, Fluka, Switzerland) and $150 \mathrm{mM}$ sodium chloride (CAS RN: 7647-14-5, Roth, Germany) in ultrapure water filtered through Milli-Q Gradient A10 filters (Millipore AG, Switzerland) having 18.2 $\mathrm{M} \Omega \cdot \mathrm{cm}$ at $25{ }^{\circ} \mathrm{C}$. The $\mathrm{pH}$ was adjusted to 7.4 using $6 \mathrm{M} \mathrm{NaOH}(\mathrm{CAS} \mathrm{RN}$ : 1310-73-2, Sigma-Aldrich Chemie GmbH, Switzerland).

\section{TRCDA / DOPC vesicle preparation}

10,12-Tricosadiynoic acid (TRCDA, CAS RN: 66990-30-5, Sigma-Aldrich, USA and Alfa Aesar, USA) was stored as follows. First, the powder was dissolved in chloroform, aliquoted, dried by a stream of nitrogen, and then resuspended in cyclohexane containing $4 \%$ ethanol. The resuspended TRCDA was cooled down with dry ice for 1 hour and lyophilized for 72 hours to remove cyclohexane/ethanol. This procedure minimizes unwanted crosslinking during the storage. The vials were filled with nitrogen (Nitrogen 4.5, PanGas, Lot. 756 311-00179757, $\mathrm{CH})$ and stored in a freezer until use $\left(T=-26^{\circ} \mathrm{C}\right)$. Prior to use the powder was resuspended with chloroform. Once TRCDA was dissolved in chloroform, the aliquot was used within 4 months. 1,2-dioleoyl-sn-glycero-3-phosphocholine (DOPC, CAS RN: 4235-95-4, Avanti Polar Lipids, USA) and 1,2-dioleoyl-sn-glycero-3-phosphoethanolamine-N-(7-nitro-2-1,3benzoxadiazol-4-yl) (ammonium salt) (NDB-PE, CAS RN: 384823-46-5, Avanti Polar Lipids, USA) stored in chloroform were used as received. Depending on the experiment, different molar ratios of TRCDA, DOPC and NDB-PE were mixed in a glass vial. The molar ratio for the samples presented in the section of "TRCDA/DOPC sample preparation" was 50:50:1 and 1:1:0 for the samples used in Figure 1B,D-G and Figure 3 in the main text. After the evaporation of chloroform under a nitrogen stream, the vial was kept under vacuum at 400 mbar for at least 14 hours to completely remove the solvent residuals, followed by rehydration with HEPES buffer solution at a total lipid concentration of $1 \mathrm{mM}$, vortex for $t=30 \mathrm{~s}$, and high-power probe sonication (Omni Sonic Ruptor 400 Ultrasonic Homogenizer, Omni International, USA) for 30 min in a water bath at $T=65{ }^{\circ} \mathrm{C}$. In order to remove titanium particles introduced by the probe sonication process, the TRCDA suspension was either sterile filtrated through a $0.22 \mu \mathrm{m}$ filter 
or centrifuged by the Sorvall Legend X1R (Thermo Fisher scientific, USA) in a conical falcon tube for 5 minutes at $1000 \mathrm{rpm}$. The segregated titanium particles were visible at the bottom. Only the supernatant was used for the rest of the experiments. The second method was applied for ITC and UV-Vis measurements, whereas the first was used for the rest of the experiments. The suspension was then stored at $T=2{ }^{\circ} \mathrm{C}$ for at least 12 hours in order to give enough time for the diacetylene to self-assemble (see also "TRCDA/DOPC sample preparation").

\section{Polymerization}

TRCDA monomer was polymerized either by a UV box (CL-1000 crosslinker, UVP, USA) or a UV hand light (NU-4 KL, Konrad Benda, Germany) with a UV dose of $0.1 \mathrm{~J} / \mathrm{cm}^{2}$ for supported crystals and $0.2 \mathrm{~J} / \mathrm{cm}^{2}$ for suspensions.

\section{Peptide preparation}

Melittin from honey bee venom (CAS RN: 20449-79-0, GenScript, USA), polymyxin B (CAS RN: 1405-20-5, Sigma-Aldrich, USA), polymyxin E (CAS RN: 1264-72-8, Sigma-Aldrich, USA) and magainin 2 (CAS RN: 108433-95-0, Biobyt Ltd, UK) were aliquoted in HEPES buffer solution at different concentrations and stored in a freezer $\left(T=-26^{\circ} \mathrm{C}\right)$ until use. Once the stock solutions were thawed, they were kept in a fridge $\left(T=2{ }^{\circ} \mathrm{C}\right)$ and used within a couple of days.

\section{UV-Vis spectroscopy}

The measurements for the experiments in Figure S1 were carried out with a UV-Visible Spectrophotometer (CARY 50 Scan, Varian/Agilent USA) using a $1 \mathrm{~cm}$ path length cell (High Precicion Cell, Hellma Analytics, Germany). The melittin solution was added to the vial containing the polymerized TRCDA/DOPC suspension with a plastic pipette and then gently agitated to mix the suspension. The peptide-to-lipid ratio was 0.03 . The incubation time was 30 minutes. The scan rate, data interval, averaging time were $300 \mathrm{~nm} / \mathrm{min}, 0.5 \mathrm{~nm}$, and $0.1 \mathrm{~s}$ respectively. The measurements for the experiments in Figure 1A and 2A were carried out with a microplate reader (Synergy H1, BioTek, USA) in the range of 300-800 $\mathrm{nm}$ and $1 \mathrm{~nm}$ spacing. The TRCDA suspension was prepared as described above, distributed into a 96 well-plate and polymerized by the CL-1000 crosslinker at $0.2 \mathrm{~J} / \mathrm{cm}^{2}$. The peptide solution was added and the plate was shaken for 15 minutes with an orbital shaker (Orbi-ShakerTM JR., Benchmark Scientific, USA) at $200 \mathrm{rpm}$. The total incubation time was 1 hour. Besides the subtraction of the baseline (HEPES buffer solution), Rayleigh scattering was either removed by fitting the 
data points with the function $f(x, a, b)=\log \frac{1}{\left(1-1 \cdot 10^{a \cdot x^{(-4)}}\right)}+b$ by freeing $a$ and $b$ over the range of $210-1100 \mathrm{~nm}$ while skipping the region $400-900 \mathrm{~nm}$, where PDA signals present (S1) or a manual subtraction via fityk was performed. Data post processing was done by LibreOffice Calc v6.3.5.2 30, gnuplot v5.2 and fityk 1.3.1.

\section{Calculation of colorimetric response}

Colorimetric response (CR) is a parameter commonly used for quantifying the chromism in PDA as follows ${ }^{1}$ :

$$
C R=\frac{R A_{0}-R A_{1}}{R A_{0}} \times 100
$$

where the relative intensity $R A_{i}$ is defined as

$$
R A_{i}=\frac{A_{i}^{\text {blue }}}{A_{i}^{\text {blue }}+A_{i}^{\text {red }}}
$$

Polydiacetylene (PDA) has two dominant peaks in the absorption spectra, which are responsible for its blue and red color. $A^{\text {blue }}$ and $A^{\text {red }}$ are the absorbances at the wavelength $\lambda_{\text {blue }}$ $=645 \mathrm{~nm}$ and $\lambda_{\text {red }}=546 \mathrm{~nm}$ respectively. The index $i=[0,1]$ refers to the absorbance before and after inducing the transition.

\section{TRCDA/DOPC sample preparation for Figure $1 B, D-G$ and Figure 3}

Glass coverslips were treated with an oxygen plasma cleaner (100 Plasma System, PVA TePla, Germany) and incubated with a hot $\left(T=65^{\circ} \mathrm{C}\right)$ vesicle suspension (preparation described above) for $15 \mathrm{~min}$ on the bench, during which the samples cooled down to room temperature $\left(T \approx 23{ }^{\circ} \mathrm{C}\right)$. These samples rested further in a fridge $\left(T=2{ }^{\circ} \mathrm{C}\right)$ at least for 12 hours and then were rinsed with HEPES buffer solution. This resting time is important for the crystal domain formation. Donut-shaped PDMS (Sylgard 184 silicone elastomer kit, Dow Corning, USA) blocks were used to confine the suspension on the glass coverslips. Polymerization was carried out by the UV box as described above. 

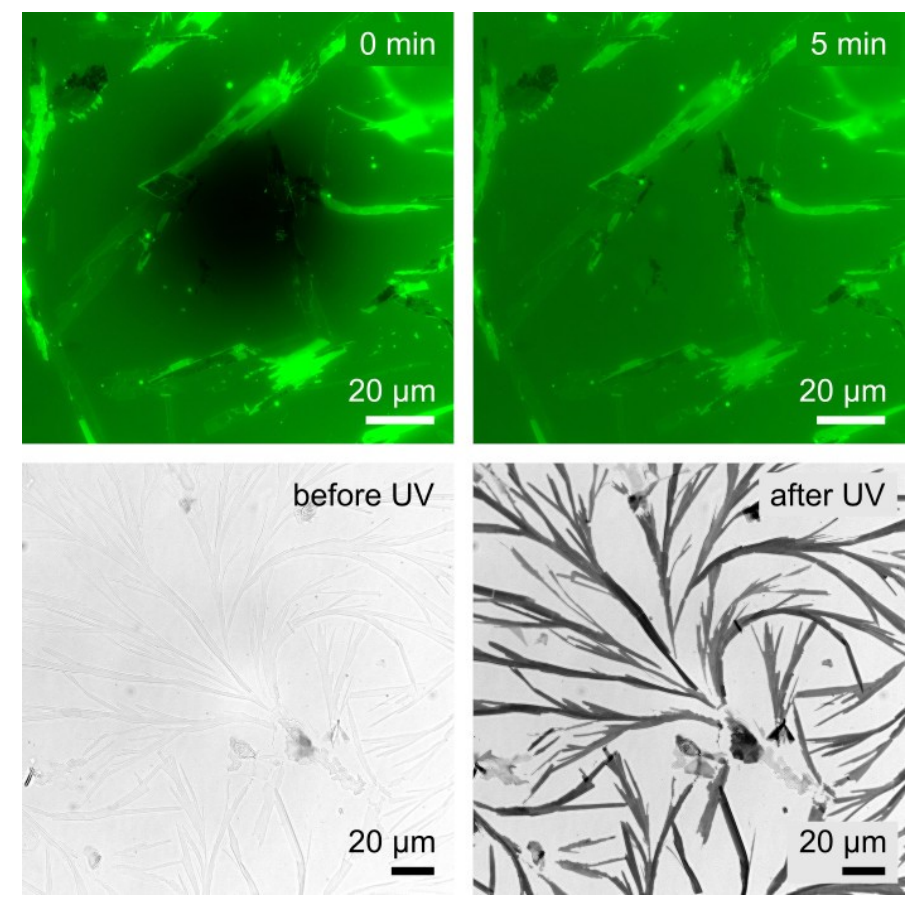

The formed structure on glass consists of fluidic DOPC bilayers, as seen by the fluorescence recovery after photobleaching of NBD-PE mixed at $1 \mathrm{~mol} \%$, and TRCDA domain structures

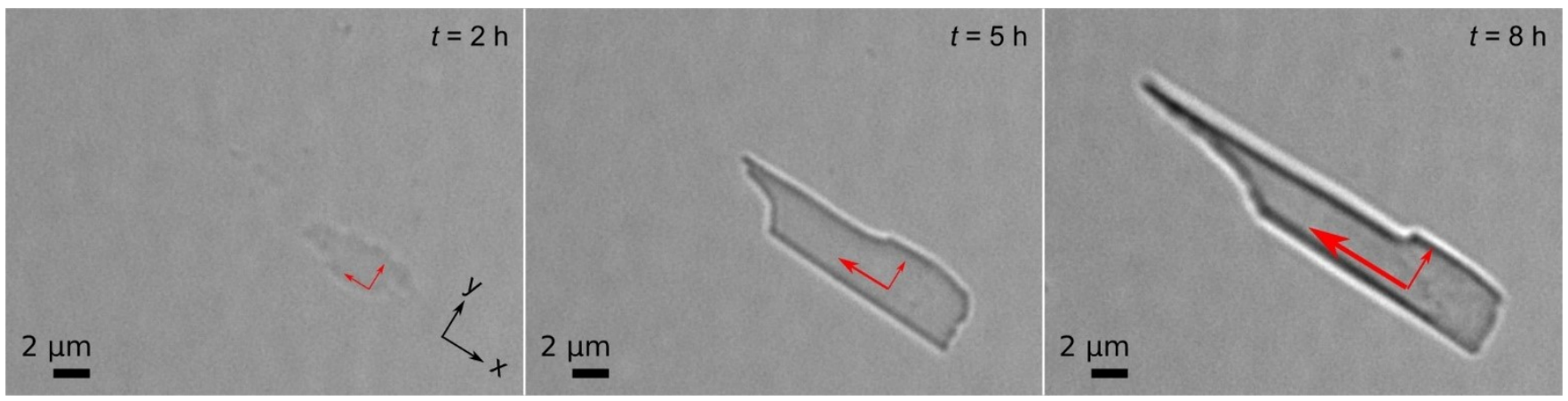

that are visible also in bright field microscopy (see the above microscopy images). These TRCDA domains grew anisotropic (grew much faster in one direction of the crystal than the other, see snapshots below from a bright field movie), resulting in a tree-like structure with branches. When TRCDA domains are polymerized, they form blue PDA. 

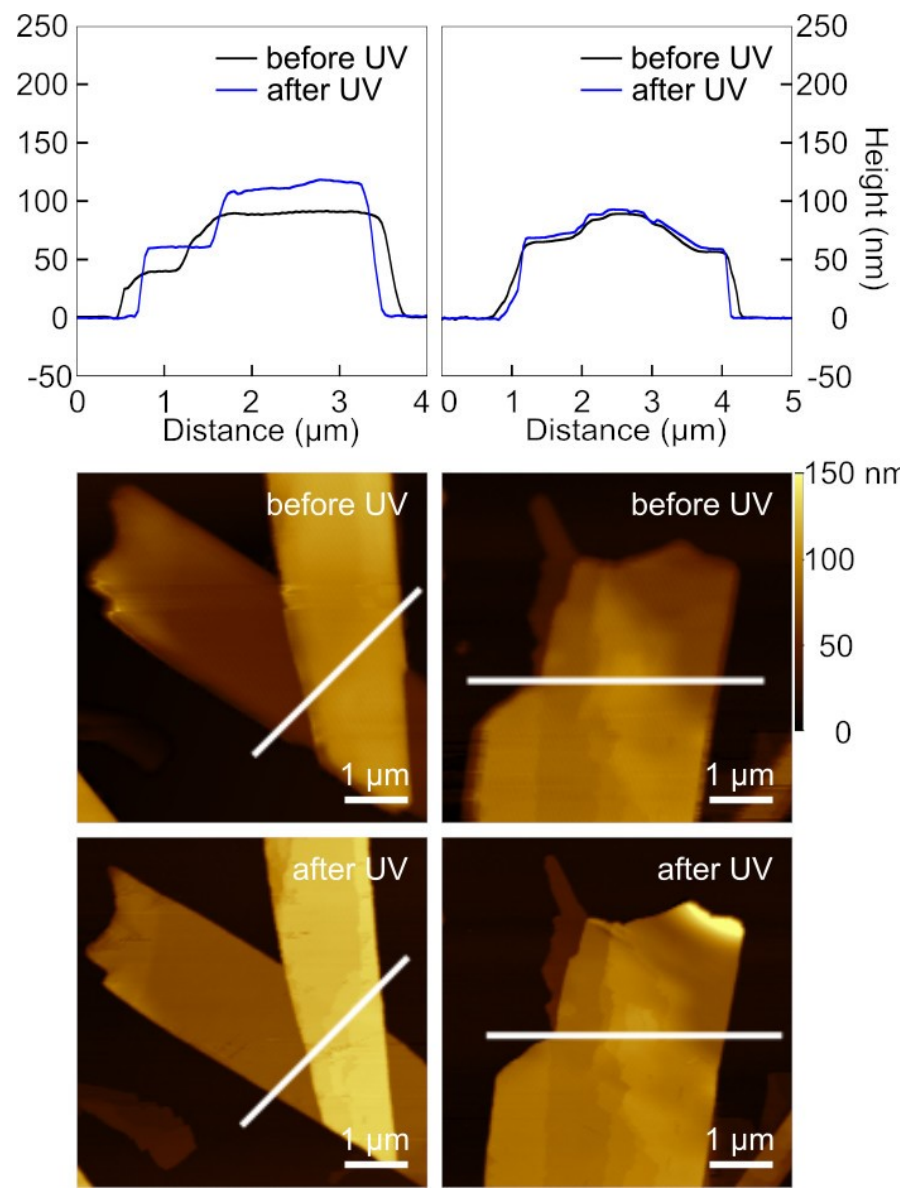

Atomic force microscopy (AFM) measurements revealed that these TRCDA domains are multilayers with a height up to $100 \mathrm{~nm}$ (see the AFM image above), which roughly corresponds to 40 monolayers, supposing $2.5 \mathrm{~nm}$ for a single monolayer thickness. ${ }^{2}$ The formation of supported lipid bilayers with TRCDA is not trivial, because the integration of diacetylene in lipid mono-/bilayers is notoriously difficult as they prefer non-lamellar phases such as helix and tubes. ${ }^{3}$ When a TRCDA monolayer assembled at a water-air interface was compressed, TRCDA protrudes from the layer, forming a segregation or a multilayer structure. ${ }^{4}$ These TRCDA multilayer domains slightly alter their shapes during polymerization, detected by AFM (see above).

Many TRCDA structures increase in their height by 5-33\%, while the width tends to become narrower after the polymerization by $8-13 \%$. This contraction of TRCDA in the direction parallel to the backbone during the polymerization has been previously reported in other AFM studies for mono- and trilayers, ${ }^{5,6}$ and also in the investigations by infrared (IR) spectroscopy ${ }^{7}$, 8 and electron diffraction analysis. ${ }^{6}$ This phenomenon is explained by the formation of the conjugated backbone, where the bond length that connects two monomer units is shorter compared to the original monomer-monomer distance set by the hydrogen bonds at the 
TRCDA head group. ${ }^{6}$ In addition, the angle of the monomers relative to the substrate is known to change during the polymerization, ${ }^{5,7}$ which could have affected the thickness and the width of the PDA.

After the formation of the polymer, in Figure 1B,D-G and Figure 3A,B melittin was added directly to the solution at $30 \mu \mathrm{M}$.
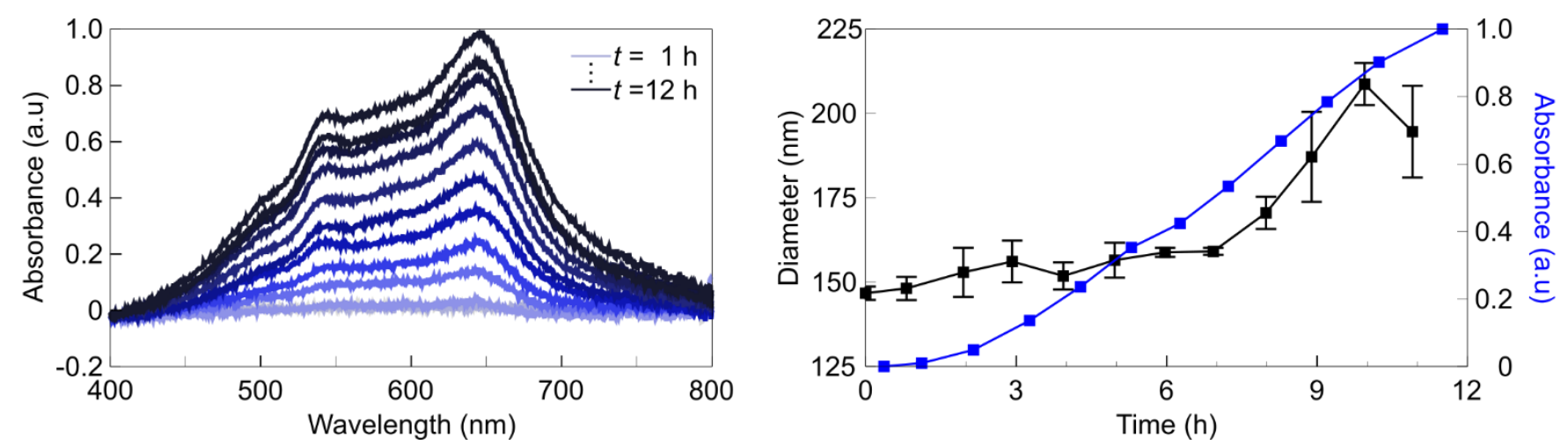

A crystal growth occurs also in PDA suspension. Tracking the particle size of assembling monomers via dynamic light scattering (DLS) reveals an increase over time. When these samples are polymerized and the absorbance is quantified via UV-Vis spectra, the intensity of the blue polymers is also enhanced in a time-dependent manner (see above).

\section{TRCDA sample preparation for Figure 1H,I}

A $1 \mu 1$ droplet of TRCDA dissolved in chloroform $(100 \mathrm{mM})$ was placed on a cleaned glass coverslip at room temperature. After the chloroform was evaporated, another $1 \mu 1$ droplet of chloroform was added on top of the dry out TRCDA. This procedure was repeated till a homogeneous film formed. The coverslip was then placed into a liquid cell (BioCell ${ }^{\mathrm{TM}}$, JPK Instruments, Germany) and filled with the buffer solution. This liquid cell allows temperature control (Figure 1I). For Figure 1H melittin was added to match a molar ratio (P/L) of 0.03.

\section{Bright field and fluorescence microscopy}

Both bright field and fluorescent images were recorded by the Eclipse Ti (Nikon, JP) with an oil immersion objective (Objective Plan-Apochromat 60x/1.40 Oil DIC, Nikon, JP), equipped with either a monochrome digital camera (DS-Qi2, Nikon, JP) or a color camera (DS-Ri2, Nikon, JP) with a resolution of 16.25 megapixel. For the bright field microscopy, a white light LED (CoolLED pE-100, UK) was used as a light source. For the fluorescence microscopy, a solid-state white light excitation source (Sola SE 5, lumencor, USA) was coupled with filter 
cubes (TRITC: 543 nm/emission, FITC: 482 nm/emission, Semrock BrightLine, USA). Images were post processed by Nikon NIS Elements v4.51.01 and ImageJ v1.52i. For the images recorded by the color camera, a plugin for Imagej was written to be able to analyze the data. The contrast and the brightness were adjusted and the fluorescent images were presented with false colors for the figures.

\section{Atomic force microscopy (AFM)}

AFM imaging were performed by the NanoWizard 3 (JPK Instruments, Germany) in either Qi mode (Figure 3D,S2A), which collects force-distance curves for each pixel, or in tapping mode (Figure 1B). For both methods gold coated n-type silicon cantilevers were employed (HQ:NSC15/Cr-Au, Mikromasch, Bulgaria and MLCT type B, Bruker, USA). The AFM resides on an active vibration isolation platform (halcyonics_i4, Accurion, Germany) in an acoustic enclosure (JPK Instruments, Germany). The setpoint and the tip velocity were in the range of $250 \mathrm{pN}$ and $8-15 \mu \mathrm{m} / \mathrm{s}$ respectively. The samples were inserted into a liquid cell BioCell (BioCell ${ }^{\mathrm{TM}}$, JPK Instruments, Germany). Data post processing was done by Gwyddion 2.55 .

\section{Isothermal titration calorimetry}

Isothermal titration calorimetry (ITC) measurements were carried out with the MicroCal iTC $_{200}$ (Malvern, UK). The TRCDA suspension was prepared as described above with a lipid concentration of 0.1 or $0.2 \mathrm{mM}$. The solution was stored for at least 12 hours at $T=2^{\circ} \mathrm{C}$. The TRCDA suspension was placed into the sample chamber with a volume of $200 \mu 1$, while the reference chamber was filled with MiliQ. The syringe was filled with $25 \mu 1$ of different peptide solutions (melittin, polymyxin B, polymyxin E, magainin 2) at different concentrations. 11 injections were carried out with a volume of $2 \mu 1$ each, a duration of $4 \mathrm{~s}$ and an interval of 300 $\mathrm{s}$ at $T=25^{\circ} \mathrm{C}$. The reference power was set to $6 \mu \mathrm{cal} / \mathrm{s}$ and the syringe stirring to $1000 \mathrm{rpm}$. Data post processing was done by Origin 7 SR4 v7.0522, LibreOffice Calc v6.3.5.2 30 and gnuplot v5.2.

\section{Dynamic light scattering}

The DLS experiment was performed by Zetasizer Nano ZS (Malvern Panalytical, UK) in the $173^{\circ}$ backscatter mode. A TRCDA/DOPC solution (1:1) was prepared as described and stored at $T=2{ }^{\circ} \mathrm{C}$. Every hour, a part of the stock solution was measured 3 times at $T=25^{\circ} \mathrm{C}$ to obtain an average value and the standard deviation. The same sample was then polymerized $\left(0.2 \mathrm{~J} / \mathrm{cm}^{2}\right)$ 
and UV-Vis spectra were recorded to obtain corresponding absorption . The raw data was post processed by LibreOffice Calc v6.3.6.2 and gnuplot v5.2.

\section{Differential scanning calorimetry}

The DSC experiment in Figure S3 was performed by the DSC 1 (Mettler Toledo, USA). $20 \mu 1$ TRCDA dissolved in Chloroform at a concentration of $\mathrm{c}=50 \mathrm{mM}$ was injected into an aluminum crucible of $40 \mu 1$ volume and dried out in a desiccator for 1 day. HEPES buffer solution either with or without melittin dissolved in it was added to the dried-out monomer and dried-out again. The final peptide lipid molar ratio was $\sim 0.15$. A range from $25-100{ }^{\circ} \mathrm{C}$ with a ramp of $5{ }^{\circ} \mathrm{C} / \mathrm{min}$ were cycled 3 times. The experiment was performed in nitrogen at a natural room pressure. The raw data was post processed by fityk 1.3.1, LibreOffice Calc v6.3.6.2 and gnuplot v5.2. 


\section{Supporting Figures}

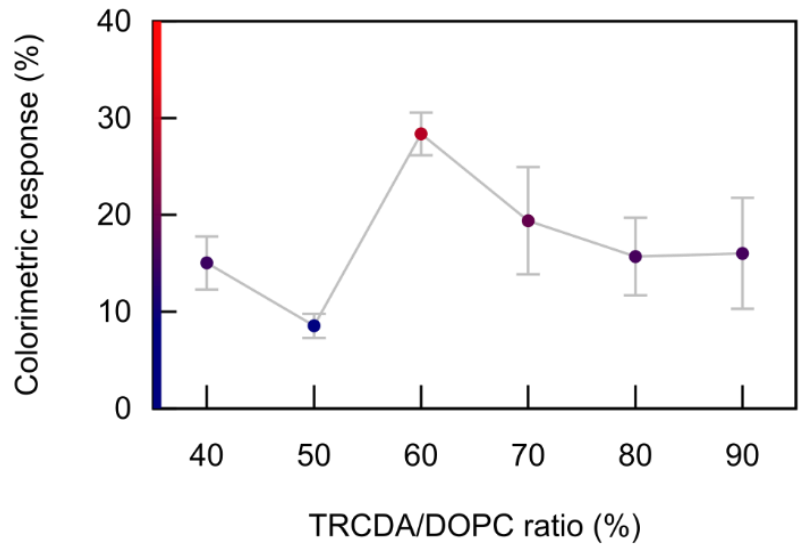

Figure S1. TRCDA/DOPC ratio dependency on the colorimetric response upon adding melittin at a peptide-to-lipid molar ratio of 0.03 .
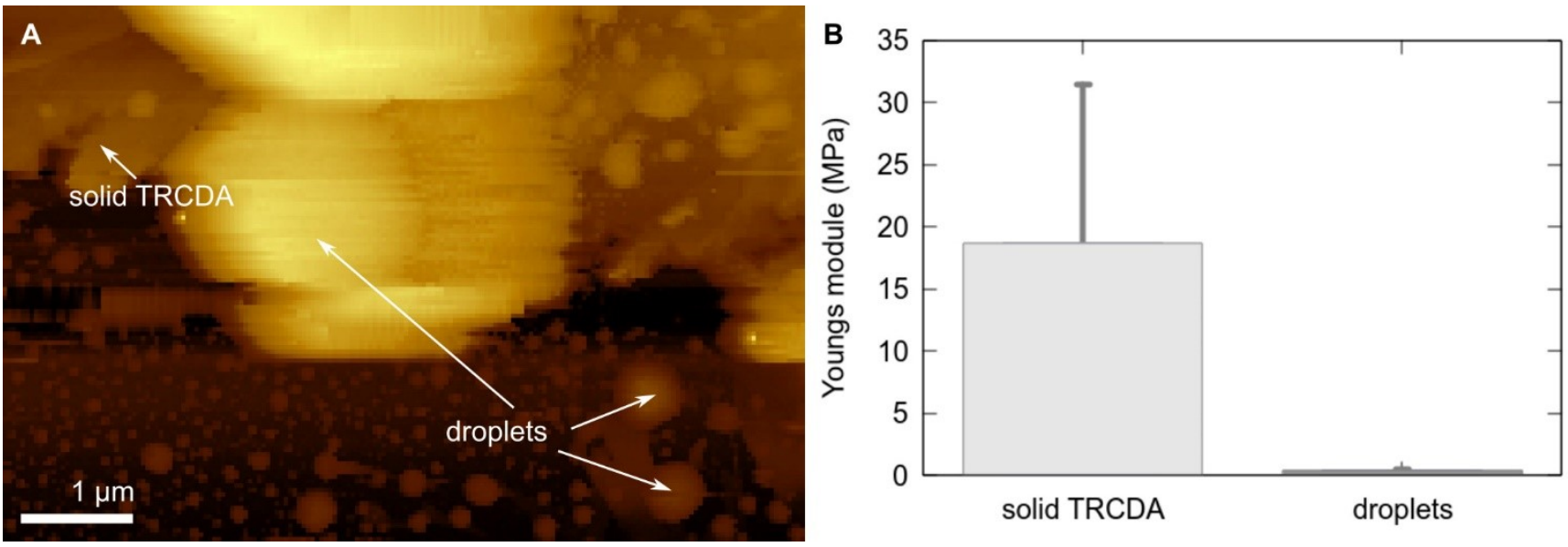

Figure S2. (A) Atomic force microscopy of monomeric TRCDA in HEPES buffer solution after adding melittin. Amorphous and crystalline structures are indicated by the arrows. Via force spectroscopy the Young's moduli for the different areas were determined (B). The Youngs modulus of these droplets are $414 \pm 126 \mathrm{kPa}$, which is comparable to soft gelatin and too soft for solid-state TRCDA. This supports that the "droplet" is TRCDA in a liquid state. 


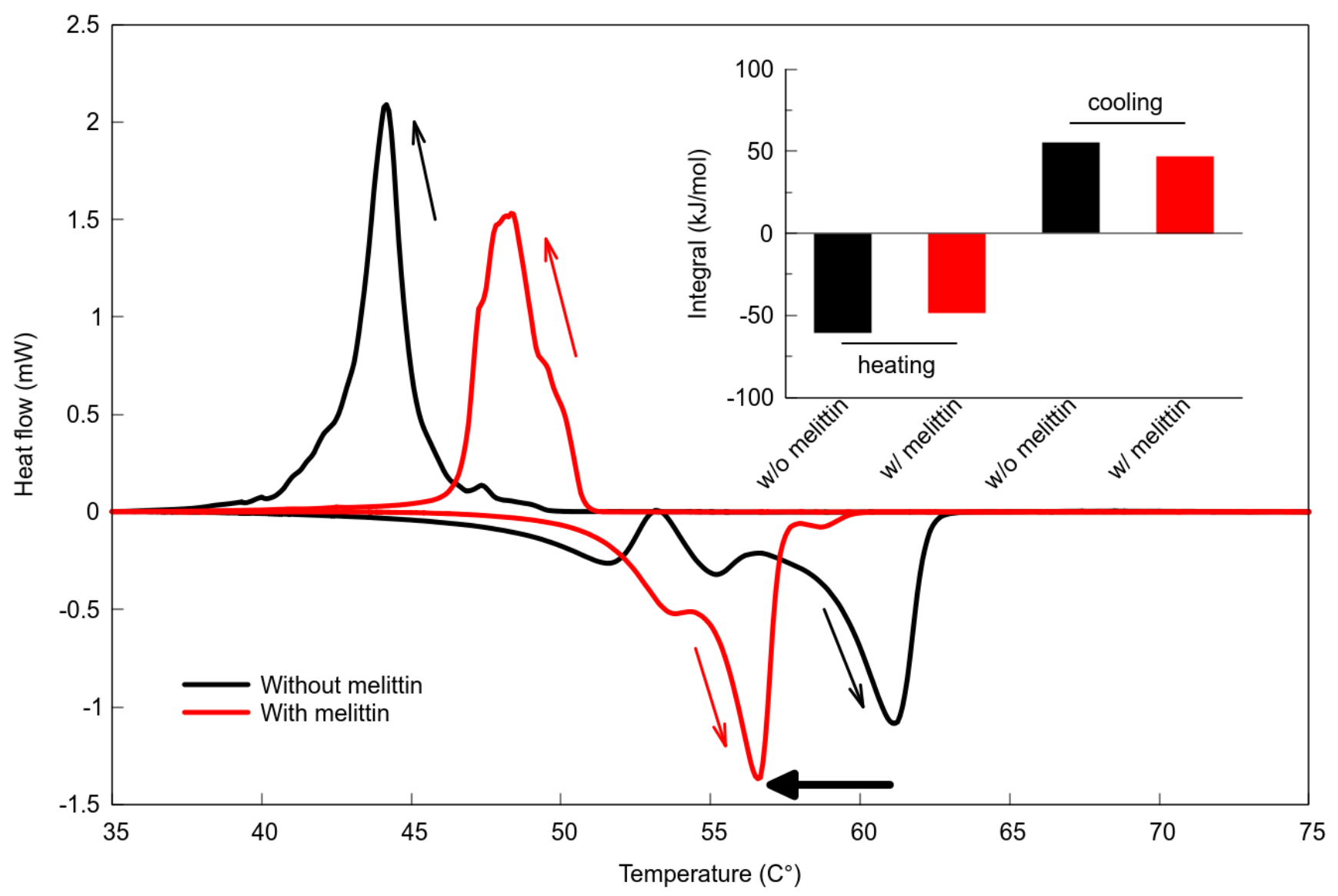

Figure S3. Differential scanning calorimetry of TRCDA monomer with and without melittin at a $(\mathrm{P} / \mathrm{L})$ ratio of $\sim 0.15$. In case of the presence of melittin, the transition temperature decreased by $\sim 4.4^{\circ} \mathrm{C}$ while heating. The inset shows the integrated (total) normalized heat transferred at the transitions. The total heat for the phase transition is reduced by $\sim 20 \%$ with melittin. This is because the addition of melittin had partially transformed the solid TRCDA into liquid, thus less solid-state TRCDA was left in the chamber for the further phase transition when the temperature was being scanned. Both suggest that melittin triggers the solid-to-liquid transformation of TRCDA. Interestingly the crystallization temperature increased when melittin was added, presented by the peak temperature shift from $44.2{ }^{\circ} \mathrm{C}$ to $48.3{ }^{\circ} \mathrm{C}$ during cooling. A possible explanation is that the addition of melittin acted as an impurity, which may have helped with the solidification as more nucleation centers were available compared to pure TRCDA. Note that the signals with melittin contain salts in the chamber due to the $\mathrm{NaCl}$ in the melittin solution, yet the control experiments confirmed that salts yield no significant signals. 

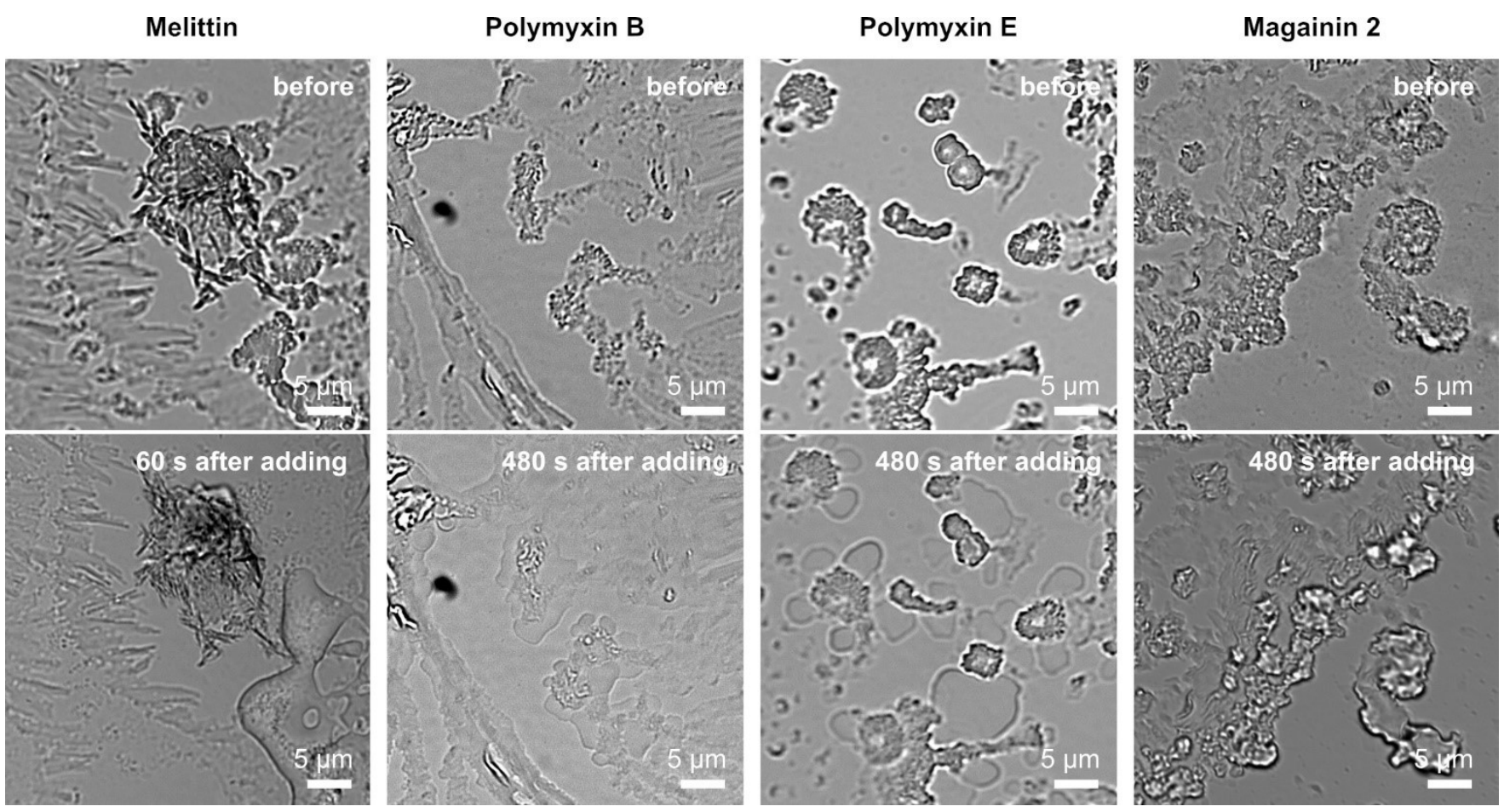

Figure S4. Bright field microscopy image of N-[2-(ethylamino)ethyl]-10,12-pentacosadiyneamide hydrogen bromide assembled on a glass coverslip before and after addition of different peptides. The concentration of the added solution was $1 \mathrm{mM}$ for melittin and $2 \mathrm{mM}$ for polymyxin B, polymyxin E and magainin 2.

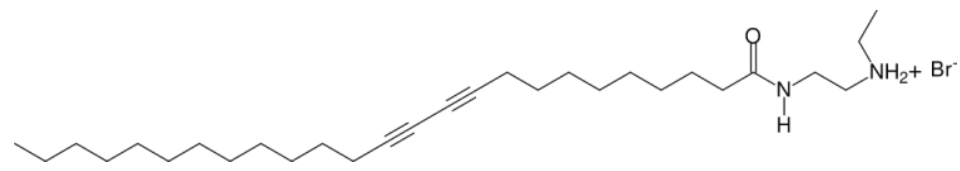

Chemical structre of Poly(N-[2-(ethylamino)ethyl]-10,12-

pentacosadiyneamide hydrogen bromide 
Polymyxin B
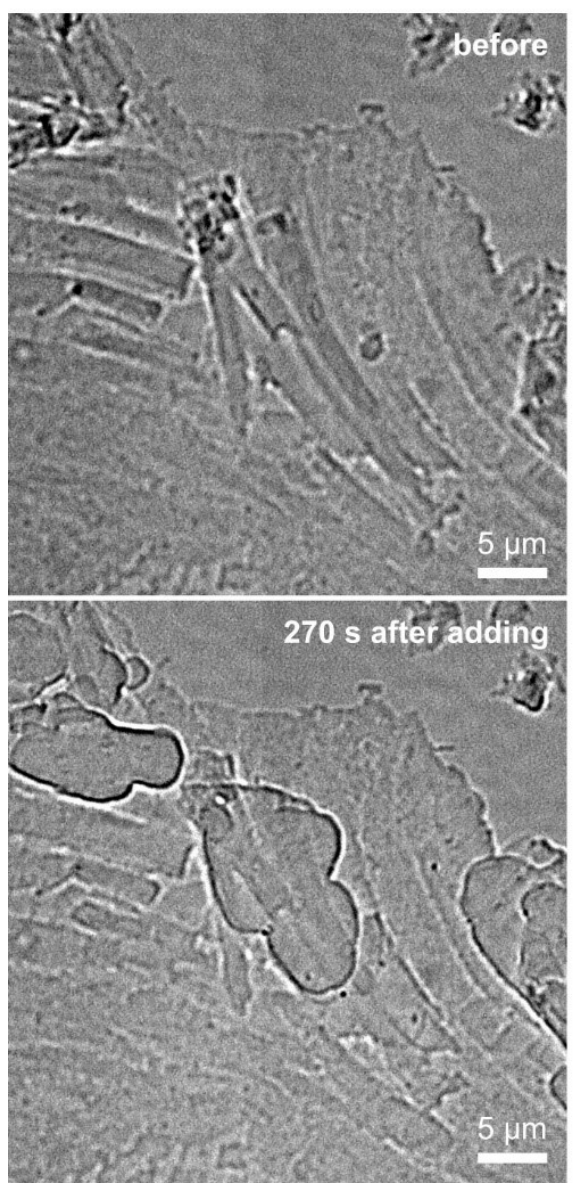

Polymyxin E
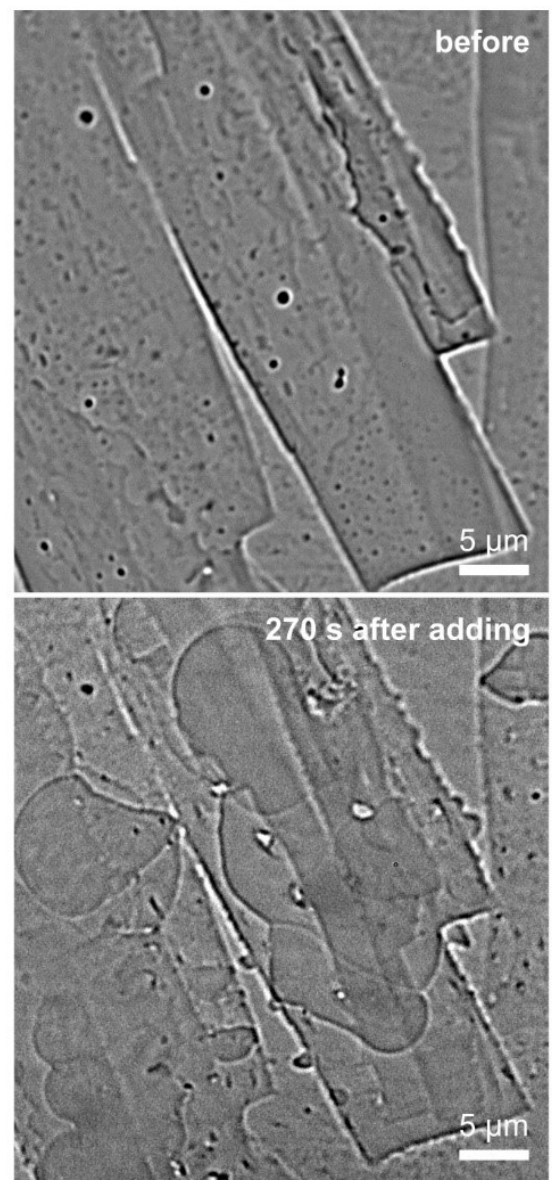

Magainin 2
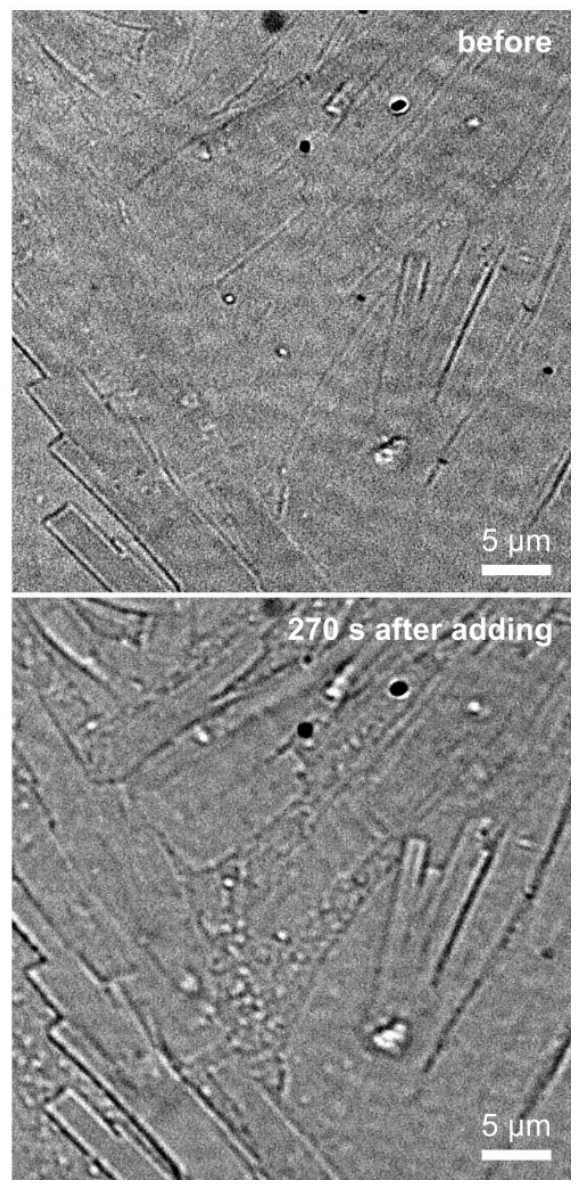

Figure S5. Bright field microscopy images of TRCDA monomers assembled on a glass coverslip before and after addition of different peptides (polymyxin B, E and magainin 2) at a molar ratio (P/L) of 10. 


\section{REFERENCES}

1. Charych, D. H.; Nagy, J. O.; Spevak, W.; Bednarski, M. D., Direct colorimetric detection of a receptor-ligand interaction by a polymerized bilayer assembly. Science 1993, 261 (5121), 585-8.

2. $\quad$ Carpick, R. W.; Sasaki, D. Y.; Burns, A. R., First observation of mechanochromism at the nanometer scale. Langmuir 2000, 16 (3), 1270-1278.

3. Pakhomov, S.; Hammer, R. P.; Mishra, B. K.; Thomas, B. N., Chiral tubule selfassembly from an achiral diynoic lipid. P Natl Acad Sci USA 2003, 100 (6), 3040-3042.

4. Volinsky, R.; Kolusheva, S.; Berman, A.; Jefinek, R., Investigations of antimicrobial peptides in planar film systems. Bba-Biomembranes 2006, 1758 (9), 1393-1407.

5. Sasaki, D. Y.; Carpick, R. W.; Burns, A. R., High molecular orientation in mono- and trilayer polydiacetylene films imaged by atomic force microscopy. J Colloid Interf Sci 2000, 229 (2), 490-496.

6. Kuriyama, K.; Kikuchi, H.; Kajiyama, T., Molecular packings - Photopolymerization behavior relationship of diacetylene Langmuir-Blodgett films. Langmuir 1996, 12 (26), 64686472 .

7. Saito, A.; Urai, Y.; Itoh, K., Infrared and resonance Raman spectroscopic study on the photopolymerization process of the Langmuir-Blodgett films of a diacetylene monocarboxylic acid, 10,12-pentacosadiynoic acid. Langmuir 1996, 12 (16), 3938-3944.

8. Ortuso, R. D.; Ricardi, N.; Burgi, T.; Wesolowski, T. A.; Sugihara, K., The deconvolution analysis of ATR-FTIR spectra of diacetylene during UV exposure. Spectrochim Acta A 2019, 219, 23-32. 